\title{
EL PAPEL DE LA MATEMATICA EN EL PROCESO EDUCATIVO INICIAL
}

\author{
DE GUZMAN, M. \\ Catedrático de la Universidad Autónoma de Madrid
}

\section{SUMMARY}

A short analysis of the educational values of Mathematics and of the main defects that can be observed in the current trends in Spanish primary and secondary schools, together with some remedial suggestions.

\section{EDUCACION COMO TRANSMISION DE CULTURA}

Educación es desarrollo integral de la persona dentro de un determinado contexto cultural y social. Comporta por tanto un proceso de transmisión de una cultura y, al tiempo, una preparación para la posible superación de la Cultura transmitida.

La cultura connota un sinfin de componentes no fácilmente definibles, pero entre ellas se pueden destacar como fundamentales la actividad intelectual y la recep. tividad a la belleza y sensibilidad humanas, como afirma Whitehead, (1917). Estas características están necesariamente sumergidas en un momento histórico y en un ambiente geográfico y social determinados. Se trata de elegir convenientemente los elementos que tales circunstancias concretas of recen para iniciar a las generaciones jóvenes en el ejercicio de la cultura.

Desde el punto de vista formal, la actividad intelectual conlleva un contenido sobre el que se ejercita y un estilo adecuado de ejercitarse. De cada uno de los aspectos de la cultura que nuestra sociedad juzga merecedores de perperuarse por su fecundidad, utilidad, belleza, etc... es necesario seleccionar cuidadosamente aquellos que más adecuadamente representan los va. lores a transmitir.

Puesto que lo que debe transmitirse no son meros contenidos, sino un espíritu de actividad intelectual, el estilo adecuado de transmisión será el que más eficazmente estimule la propia actividad. No interesa en $a b$. soluto transmitir muchos conocimientos, sino transmitir much a capacidad de actividad intelectual alrededor de unos pocos conocimientos representativos. Puesto que se pretende transmitir tambien una capacidad de superación de la cultura heredada, es importante transmitir una comprensión en profundidad que engendre lo actividad del espiritu critico acerca de su propio saber.
La formación para la receptividad a la belleza requie. re tener bien claro el tipo y caracten'sticas de belleza que puedan encontrarse en el objeto en que nos ejercitamos. Será pues necesario escoger aspectos de este objeto en que mejor respiandezca la belleza propia de él. Aquellos aspectos ante los que el educador mismo es capaz de vibrar con más entusiasmo estético.

La apertura a la sensibilidad humana puede ser fomentada resaltando los elementos que el objeto en cuestión presenta con resonancias en el resto de la actitud profunda del hombre. La personalidad de los hombres que se han ejercitado con más entusiasmo e inspiración en èl. La génesis y evolución de las ideas importantes, el entronque con la historia de la sociedad que les ha dado origen, etc... son aspectos que deberiamos subra. yar en el proceso de transmisión de los diferentes as. pectos importantes de nuestra cultura.

\section{LA PRESENCIA DE LA MATEMATICA EN EL PROCESO EDUCTIVO}

La presencia vigorosa de la matemática en el proceso educativo es algo que nadie discute hoy seriamente. Los aspectos más característicos del pensamiento occiden. tal, su filosofía, su tecnología, están íntimamente ligados al pensamiento maternático, de forma especialmente profunda a partir del siglo VI a. de C. con los pitagóricos. Los momentos más pujantes de la cultura de occidente coinciden con los momentos de cultivo más intenso de la matemática, arrastrando consigo las otras ciencias en su desarrollo y propiciando la evolución tecnológica.

La matemática ha sido y es un saber extraordinaria. mente polivalente y como tal presenta caracteristicas que la hacen extraordinariamente adecuada para la 
transmisión de las capacidades propias de nuestra cultura. La matemática es a la vez:

a) Una ciencia con sus fines propios. Entre ellos la ordenación racional y lógica de los aspectos cuantitativos, en sentido amplio, de las estructuras reales y mentales.

b) Un arte, que consigue, al menos como premio añadido a su esfuerzo por alcanzar sus objetivos especificos, la creación de estructuras mentales profundamente bellas.

c) Un instrumento poderoso de exploración y transformación del universo.

La múltiple aportación de la matemática al proceso educativo es así extraordinariamente valiosa y no es extraño en absoluto que haya sido desde los pitagóricos hasta nuestros días, pasando por el cuadrivio de la edad media, uno de los ejes fundamentales de la educación.

A lo largo de la historia, especialmente durante los ú\}timos cuatro siglos, la sociedad que ha descuidado el cultivo activo de la matemática y de la ciencia ha visto sus potencialidades extraordinariamente mermadas. Este ha sido en particular el caso de la nuestra, cuya creatividad matemática y cientifica ha ocupado un lugar $\tan$ bajo entre las diferentes sociedades de nuestro continente.

\section{ALGUNOS DEFECTOS DE NUESTRA EN- SEÑANZA MATEMATICA}

A nivel mundial se ha dado una ciara y fuerte crisis en la educación matemática elemental. Muchos paises ya han tomado las medidas correctivas adecuadas. En el nuestro aún estamos padeciendo las consecuencias. El examen de los defectos principales que se pueden detectar en nuestro sistema educativo (Guzmán, 1983) puede darnos luz para tratar de salir de ellos y no volver a cometerlos.

En la enseñanza básica de nuestros alumnos se observà un aglomerado extraño. Unos rudimentos de teoria de conjuntos, que vienen a constituir unos cuantos acertijos aislados cuya relación con ia matemática tal vez consista para los niños en que se pueden expresar con unas palabras mágicas que además tienen su traducción cabalística en simbolos misteriosos. Una iniciación a otra familia de palabras como grupo, elemento nestro, inverso, anillo..., que se les dice que son entes muy importantes, aunque no se les explique muy bien qué se puede hacer con ellos. Y cuentas, que es lo que al parecer tiene algo que ver con la vida real, para averiguar que si en una granja hay veinticinco gallinas, cada una pone dos huevos diarios y la docena se vende a 96 pesetas... A rsto se anaden unas pincelacias de : tas, ángulos, curvas, despilfarrando mucha más ener. gia en nombres y distinciones (no se vaya a confundir un ángulo con una región angular) que en tratar de hacer algo interesante con los objetos que se introducen. En resumen, los defectos que, a mi parecer, aquejan más gravemente la enseñanza primaria podrian resumirse en cuanto a la forma en una notable desviación del objetivo principal de las matemáticas, que consiste en saber resolver problemas que puedan resultar ade. cuados e interesantes, en una ausencia de espiritu activo, de espiritu lúdico, de conexiones con el mundo real de los niños y sus intereses, en un énfasis excesivo y perjudicial en nombres y distinciones con merma de lo que es mucho más importante, la imagen, la intuición, los automatismos operativos útiles. En cuanto al contenido hay exceso de conjuntos, de álgebra, donde los problemas que se pueden proponer en una etapa inicial son meras tautologias y reconocimiento de nombres. Por otra parte se nota la ausencia de contenidos geométricos interesantes y de conexiones y aplicaciones a otras ciencias.

La enseñanza secundaria está afectada de un mal específico, además de adolecer de las mismas tendencias hacia el formalismo, abstracción y pasividad que enferman la primaria. En la enseñanza de BUP y COU se da además, a mi parecer, una confusión de objetivos que la convierten en una especie de minienseñanza universitaria. La enseñanza básica y la secundaria deberian tener idealmente unos objetivos plenamente diferenciados de los de la enseñanza universitaria. En aquellas, el elemento formativo debería ser claramente el esencial, debiendo estar los contenidos totalmente subordinados en su extensión y en su conformación a la finalidad formativa de estas etapas de la enseñanza. Con la enseñanza secundaria no se debe pretender impartir unos conocimientos que hagan de la personalidad del estudiante un mosaico de miniprofesionales de las diferentes materias. Se debe tratar con ella de ayudarle a conformar su personalidad intelectual mediante la asimilación profunda y activa de unas pocas ideas matrices en algunos campos de las ciencias y de las letras, asimilación realizada pausadamente, de modo vital, entroncando estas ideas matrices con la personalidad de sus descubridores, con la historia de su génesis y su evolución, con muestras de su fecundidad en nuestra cultura actual. En lugar de ello (véanse los libros de texto actuales) se ofrece al estudiante adolescente, muchas veces ávido de un contacto vital de este tipo, largas listas secas y pedantes de meros nombres o de ideas descarnadas de las que pronto se hastia por su aparente inutilidad. La matemática tiene una historia milenaria y apasionante, y constituye toda una aventura del pensamiento observar desde nuestra perspectiva los rodeos, los caliejones sin salida aparente, los túneles obscuros, las controversias de la evolución del pensamiento matemático hasta nuestro días. Por otra parte la riqueza de la personalidad de muchos de los matemáticos más eminantes es ciertamente impresionante. La importancia sociológica y cultural de la matemática ha sido grande, especialmente en ciertas etapas de la evolución cultural de Occidente. La simbiosis de las matemáticas con otras ciencias, con la tecnologia e incluso con la filosofía presenta aspectos muy interesantes que se prestan a discusión aun al nivel más 
elemental. ¿Cuántos de nuestros enseñantes hacen un esfuerzo por entreverar los conocimientos que imparten con explicaciones de este tipo?

\section{ALGUNOS MALES CONCRETOS QUE HAY QUE EVITAR}

A la vista de los defectos que actualmente sufrimos en nuestra educación matemática podemos tratar de delimitar explícitamente unos cuantos males que deberiamos tratar de evitar en una futura reestructuración de nuestra enseñanza. Estos son:

\section{a) Ideas inertes en los contenidos.}

La actividad matemática se debe centrar en unos contenidos adecuados. ¿Cuáles? El principio abstracto es claro: Aquellos contenidos que mejor estimulen el interés, la acción de los alumnos mismos a quienes se les proponen. Más adelante trataré de especificar cuáles pueden ser estos contenidos, al menos con algunos ejemplos. Ahora quisiera solamente subrayar que lo que hay que evitar a toda costa son las que Whitehead ha llamado ideas inertes. Ideas inertes son las que me. ramente se reciben en la mente, sin ser utilizadas, verificadas o introducidas en combinaciones nuevas.

Al tratar de concretar los contenidos adecuados habremos de preguntarnos constantemente: ¿Serán los alumnos capaces de hacer algo hoy mismo con esta idea, con esta técnica? ¿Podrán asimilarla ahora mismo hasta el punto de que la incorporen en su esquema mental y pueda surgir espontáneamente en su espiritu ante la situación adecuada? ¿Serán capaces de convertirla hoy en una herramienta activa que les sirva ahora mismo para resolver algún problema que realmente les pueda interesar? ¿O servirá acaso solamente para formar parte de una lista de nombres y de conceptos inútiles que les ayude solamente para responder a las preguntas vacias de un examen y para estimular su propia pedanteria? Incluso aquellos contenidos verdaderamente útiles los podemos presentar cargados de lastre conceptual inútil y perjudicial y de terminologia pedante que obscurece la idea original que en su génesis presentaba mucho más clara su propia fecundidad.

Para presentar ejemplos de ideas inertes en nuestros actuales contenidos no hay más que ojear someramente los textos ordinarios de nuestra enseñanza básica y secundaria. He aqui unos cuantos:

El tratamiento explicito de elementos de la teoría de conjunios en $E G B$ y $B U P$. ¿Acaso hablamos explicitamente a los niños de $E G B$ del principio de contradicción? El niño sabe mucho mejor lo que es un conjunto y las operaciones que con él se pueden hacer antes de que le obscurezcamos la mente con nombres pomposos y disquisiciones totalmente fuera de lugar y de tiempo. son estructuras fundamentales de la mente sobre las que no sólo es inutil, sino probablemente per. judicial lucubrar a destiempo.

Las pedantes introducciones del numero natural que se suelen encontrar en los textos de los primeros años de $E G B$. Si no se lo explican el nitro sabe lo que es un número. Después de la explicación ya no. ¿Puede hacer algo el ninto con tales explicaciones? Esta es probablemente una de las formas de originar los claros bloqueos psicológicos de tantas y tantas personas adultas inteligentes que confiesan que siempre se les han dado tan mal las matemáticas.

Las disquisiciones sobre grupos, anillos, etc. a nivel elemental. La introducción del álgebra se pensó que podria ser la fuente alternativa de introducción al pensamiento riguroso, tan dificil en la introducción de la geometria del estilo euclideo. Ha resultado ser fundamentalmente una fuente de ideas inertes, mero reconocimiento de nombres sin interés ninguno para la actividad intelectual del niño.

Las pedantes y filosóficas disquisiciones sobre magnitudes a nivel de enseñanza primaria.

Las lucubraciones academicistas alrededor de los teoremas fundamentales del análisis en la segunda enseñanza. Por ejemplo, la introducción del número complejo, estilo Bourbaki, a través de las clases de congruencia, módulo $x^{2}+1$, del conjunto de los polinomios sobre el cuerpo de los números reales ( ${ }^{\mathrm{C}} \mathrm{Curso} 1^{\circ}$ de BUP!).

\section{b) Impreparación de profesores.}

Cualquier intento de reforma de la enseñanza matemática está encaminado al fracaso de no contar con profesores capaces y bien preparados. La formación que oficialmente reciben los profesores en las Escuelas Universitarias de Profesorado de EGB es, en mi pinion, claramente insufficiente. Para impartir un conocimiento adecuado, con el espiritu formativo que he recalcado antes, habria que saberlo bien a fondo y ser capaz de saborearlo y colocarlo en su perspectiva propia. Para enseñar como 15 hay que saber como 100 y guardarse 85. La pedagogia puede ayudar a comunicar, pero con el que no sabe suficiente no ejercerá milagros. La formación que reciben nuestros licenciados en las Facultades suele estar muy sesgada hacia la investigación matemática. Incluso aquellos que deciden escoger la especialización en didactica matemática carecen oficialmente de cursos dedicados, por ejemplo, a la historia y evolución de las ideas matemáticas, a las aplicaciones didacticas de los juegos matemáticos o a los problemas clásicos de la matemática y se ven obligados en cambio a seguir cursos superespecializados que poco o nada utili les proporcionan para su futura tarea. Una ordenación más adecuada de la formación de las Escuelas Universitarias de Profesorado de EGB y de las Facultades no seria extraordinariamente dificil.

El paso por cursos de reciclaje y perfeccionamiento del profesorado deberia ser, especialmente en tiempos de cambio, no ya una posibilidad fácil de practicar, sino una verdadera obligación. ¿Cómo se puede pretender de otra forma la puesta en práctica de los cámbios pro- 
fundos que es necesario promover?

\section{c) Textos inadecuados.}

Es claro que una gran parte de los profesores de ensenanza básica y media no van a ser capaces de elaborar, ni siquiera sobre la pauta de unos programas ideales, cursos medianamente satisfactorios que los implementen. Por ello se hace clara la necesidad de los libros de textos. De los males de nuestra ensenanza actual una buena responsabilidad incumbe al espiritu predominante en la generalidad de los libros de textos existentes en la actualidad, tanto de EGB como de BUP y COU. Parecen estar escritos para responder a un cuestionario, olvidando totalmente el objetivo primordial de estas etapas de la enseñanza, la formación de la personalidad integral del alumno utilizando como medio su contacto con el mundo de la matemática. Hay en particular muchos textos de BUP y COU que han con+ fundido su orientación totalmente y se asemejan mucho más a los textos universitarios que a los textos de orientación básica y formativa que serian adecuados para esta etapa.

\section{d) Exámenes y controles que impiden un proceso ver-} daderamente formativo.

Este es un problema verdaderamente dificil de resolver. Establecemos un programa y en algún punto del proceso educativo decidimos realizar un control del grado de asimilación de dicho programa por parte de los estudiantes. Muchas veces con hondas repercusiones para los estudiantes y para los centros mismos en que se forman. No tardan en hacerse notar los efectos perniciosos. Los profesores comienzan a enseñar para cubrir el programa y para nada más, olvidando su labor más propia de sabor formativo. Los estudiantes se hacen, si pueden, con un cuestionario y miden su provecho por la capacidad de responder eficientemente a él. Los Centros se consideran eficaces si una buena parte de sus estudiantes logran calificaciones satisfactorias en los controles establecidos. Y el proceso todo queda pervertido en su raiz. El objetivo fundamental se convierte en la preparación eficiente para pasar un examen, relegando a segundo término los objetivos formativos que son más difícilmente medibles.

Tal vez una buena sugerencia podria ser someter a controles directos, no a los estudiantes mismos, sino a los profesores y los centros, inspeccionando sus progra. mas y su capacidad de desarrollarlos, y supervisando simplemente los controles de éstos sobre sus propios estudiantes.

\section{CUALES SON LOS CONTENIDOS ADE- CUADOS EN LA EDUCACION MATEMATI- CA HOY?}

Teniendo en cuenta el carácter polivalente de la matemática y los objetivos de la educación general apuntados antes, a la hora de indicar más concretamente los contenidos podemos señalar los cuatro encasillamientos siguientes.

a) Bagaje necesario. Los contenidos bajo este epigrafe deberian responder a los requisitos de equipamiento minimo que un ciudadano medio de nuestra sociedad debería poseer. A través de ellos se abre ante los ojos todo el mundo de aplicaciones potentes de las matemáticas, incluso de las más elementales. No trataré de señalar pormenorizadamente cuáles deberian ser, pe. ro no es dificil indicar con ejemplos el tipo de conocimientos que deberian estar entre ellos.

1. Técnicas operativas manuales.

2. Manejo de los instrumentos de cálculo. Calculadoras.

3. Ideas sobre ordenadores. Introducción a la informática básica.

4. Ideas geométricas fundamentales. Curvas. Construcción e interpretación de planos y mapas. Tratamiento del espacio adecuado.

5. Funciones. Tratamiento matemático de la dependencia.

6. Gráficas y su interpretación matemática.

7. Probabilidad. Significado. Estadisticas. Aplicaciones.

b) Matemática como ciencia. Los contenidos de este apartado responden a la intención de abrir el camino a los estudiantes hacia una visión profunda de la matemática, haciéndoles explorar someramente sobre el campo matemático la posibilidad de construcción racional de la ciencia. Se trata de introducirles a la actividad intelectual abierta a su propia superación. No se deberia tratar aqui de enseñarles muchos conocimientos. Se trata más bien de ponerles en situación de dominar unos pocos con los que verdaderamente puedan ejercitar su propia actividad intelectual creativa. Entre los más adecuados, a mi parecer, deben figurar:

1. Introducción a la teoria elemental de números.

2. Una introducción adecuada a la construcción racional de la geometria. (Huyendo de abstracciones y rigorismos excesivos, con fuerte apoyo en la intuición espacial y con adecuado énfasis en la construcción racional).

3. Ideas fundamentales de combinatoria. Aplicaciones.

4. Manejo de funciones sencillas. Aproximacion. Polinomios.

c) Matemática como arte y juego. Los contenidos de este sabor están destinados a ejercitar la contemplación y creación de la belleza caracteristica de las matemáticas y el espíritu y actividad lúdica alrededor de ellas. Existe una gran porción del saber matematico que ha sido motivado en su origen por el juego y el placer estético y, ciertamente, la dedicación intensa a su ciencia de una buena parte de los matemáticos de todos los tiempos ha estado y está apoyada fuertemente en esta componente artistica y lúitica de la matemática.

No es dificil escoger resultados bien asequibles de la 
teoria elemental de números (por ejemplo, la demostración de la existencia de infinitos números primos), de la geometria elemental (por ejemplo, concurrencia de las alturas de un triángulo, circulo de los nueve puntos), de la topología elemental (los 7 puentes de Königsberg, las tres granjas y los tres pozos), donde este aspecto estético y lúdico se puede hacer bien patente. El valor formativo y estético de la dominación racional de la intuición espacial se podria lograr mediante la selección adecuada de unos cuantos de estos resultados.

d) Matemática como actividad humana. Los contenidos aquí enmarcados deberían estar destinados a hacer sentir el carácter profundamente humano de la actividad matemática. Por medio de su historia, la biografia de sus hombres, los impactos sobre el pensamiento general, filosófico, sobre la historia de la sociedad.

No es necesario esperar a acumular muchos conocimientos matemáticos para poder hacer estos aspectos bien patentes. La receptividad a este tipo de conocimiento por parte de los jóvenes es enorme por las implicaciones humanas que su espíritu desea vivamente conocer. Representa un fuerte estimulo para muchos este contacto vital con la historia y las personalidades tan ricas del mundo matemático.

Es fácil ver cómo con un poco de esfuerzo y con textos adecuados se puede realizar esta labor:

1. Génesis de las ideas matemáticas barajadas en la en. señanza elemental. Historia de las ideas elementales de la matemática.

2. Biografía de matemáticos importantes.

3. Poder de las ideas matemáticas. Impactos sobre la filosofía, la sociedad, la historia.

Al distribuir estos contenidos en las cuatro secciones indicadas no quisiera ser mal interpretado. No se trata de compartimientos estancos. Un mismo conocimiento matemático puede prestarse muy bien a estos cuatro tipos de actividad intelectual en torno a él. La educa. ción procede unitariamente, no por acumulación de saberes y técnicas. Es el educador quien debe ser capaz de vibrar con cada una de las facetas de la actividad matemática y señalarlas explícitamente en el momento oportuno.

\section{EL ESTILO ADECUADO DE LA ACTIVI. DAD MATEMATICA}

La matemática es esencialmente actividad, puesto que es muy fundamentalmente método de pensamiento para resolver situaciones-problema reales y mentales. La actividad matemática se ejercita mediante el enfrentamiento con problemas adecuados al estadio del desarrollo del individuo que la practica.

Lo más importante es, por tanto, hacer y hacer de tal modo que el individuo quede capacitado para hacer de modo autónomo e incluso para ir más allà. Será necesario, por lo tanto, entender, pero sin exageraciones. Un deseo de rigor prematuro, una meticulosidad excesiva, a destiempo, un encorsetamiento rigido de la intuición pueden paralizar la actividad.

El método de enseñanza más adecuado será, por consiguiente, el que mejor estimule la actividad intelectual del individuo y este es el basado fundamentalmente en problemas y aplicaciones entroncadas en situaciones de interés para él. El método basado en problemas inieresantes estimula fuertemente al individuo para hacerse capaz de crear combinaciones nuevas con las ideas e instrumentos que ya posee. Esta es la base del progreso.

La actividad matemática debe ir acompañada con cierto énfasis, por parte del educador, en el poder, la belleza, el sentido humano y profundo de las ideas que se manejan, aprovechando para ello todas las ocasiones que la misma materia proporciona.

\section{REFERENCIAS BIBILIOGRAFICAS}

DE GUZMAN, M., 1983, Sobre la educación matemática, Revista de Occidente, vol. 26, pp. 37-48.

WHITEHEAD, A.N., 1961, The Aims of Education and Ot- her Essays, en Alfred No:th Whitehead. An Anthology Selected by F.S.C. Northrop and M.W. Gross (Mac Millan: N.Y.). 